\title{
The geometry dependence of the conductance oscilla- tions of monovalent atomic chains
}

\author{
Péter Major*1,2, Géza Tichy², József Cserti ${ }^{3}$, Víctor Manuel García-Suárez $^{4}$, \\ and Skon Sirichantaropass ${ }^{4}$ \\ ${ }^{1}$ KFKI Research Institute of Particle and Nuclear Physics of the Hungarian Academy of Sci- \\ ences, H-1121 Budapest, Konkoly-Thege u. 29-33, Hungary \\ 2 Department of Solid State Physics, Eötvös University, H-1117 Budapest, Pázmány Péter sétány \\ 1/A, Hungary \\ ${ }^{3}$ Department of Physics of Complex Systems, Eötvös University, H-1117 Budapest, Pázmány \\ Péter sétány 1/A, Hungary \\ ${ }^{4}$ Department of Physics, Lancaster University, Lancaster, LA1 4YB, UK \\ Received, revised, accepted \\ Published online
}

PACS 73.23.-b, 73.23.Ad, 73.40.Cg, 73.63.-b, 73.63.Rt

Using a tight binding model we calculate the conductance of monovalent atomic chains for different contact geometries. The leads connected to the chains are modelled as semi-infinite fcc lattices with different orientations and couplings. Our aim is twofold: To check the validity of a three-parametric conductance formula for differently oriented leads, and to investigate the geometry dependence of the conductance oscillations. We show that the character of these oscillations depends strongly on the geometry of the chain-lead coupling.

Copyright line will be provided by the publisher

\section{Introduction}

Since Ohnishi [1] et al. and Yanson et al. 22 demonstrated the existence of atomic chains, there has been a rapidly growing interest in this field [3-17]. The conductance of these chains was found to oscillate as a function of length, as it had been predicted earlier in Refs. 3, 4] and studied experimentally by e.g. Smit et al. [5]. This issue has also been investigated theoretically for alkali metals, and it was concluded that chains with odd numbers of atoms have a conductance equal to $2 e^{2} / h$, while the conductance is smaller than $2 e^{2} / h$ for the chains with even numbers of atoms [6, 7, 8, 9], (hereafter we refer to this as $e<o$ ). The opposite of this, namely $e>o$ was found in noble metals [10] and also in some calculations of alkali metals [4. The role of symmetries was also studied in Refs. [11, 12, and the authors stated that in chains with mirror symmetry and an odd numbers of atoms the conductance is exactly $2 e^{2} / h$. However, Havu et al. 13 showed for sodium wires that the oscillations may depend on the geometry. Hirose et al. 14 also pointed out that the oscillations are crucially influenced by the chain-lead coupling.

There exist different simple models that help to understand and interpret the numerical and experimental results concerning the conductance oscillations, such as in [5, 6, 10, 15, 16, but none of them is suitable to explain the transformation between $e<o$ and $e>o$ due to the geometry dependence.

In our previous work [17] we verified with the ab-initio code SMEAGOL [19, 20, 21] that a three-parameter formula can describe surprisingly well the conductance of monovalent atomic

* Corresponding Author: e-mail: majorp@complex.elte.hu, Phone: +36 20490 2722, Fax:+36 13722868 
2P. Major, G. Tichy, J. Cserti, V. M. García-Suárez, and S. Sirichantaropass: The geometry dependence of the conductance oscillati

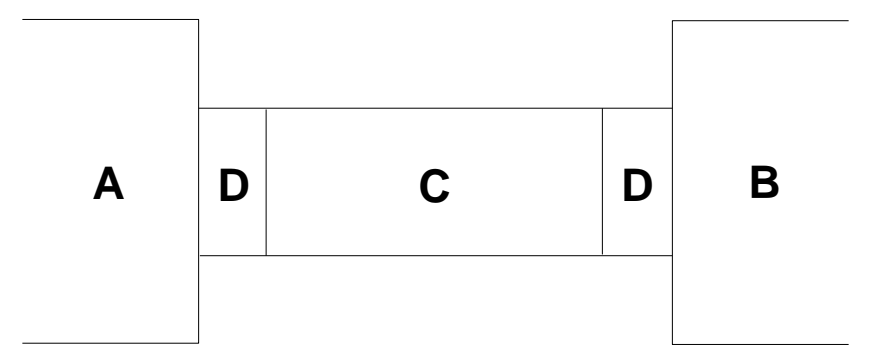

Fig. 1 The lead(A)-chain(C)-lead(B) system. The coupling atoms(D) between the chain and the leads are described by scattering matrices with reflection coefficients $r_{L}^{\prime}$ and $r_{R}$ those appear in Eq. (1).

chains, although the electron-electron interaction could destroy its applicability. In units of $2 e^{2} / h$ the conductance as the function of length is given by

$$
g=\frac{\left(1-r_{L}^{\prime 2}\right)\left(1-r_{R}^{2}\right)}{1+r_{L}^{\prime 2} r_{R}^{2}-2 r_{L}^{\prime} r_{R} \cos \left(2 k d+\Phi_{L R}\right)},
$$

where $d$ is the length of the translational invariant segment of the chain, $k$ is the wavenumber of the transmitting electron, $\Phi_{L R}=\Phi_{L}+\Phi_{R}$ and $\Phi_{L}\left(\Phi_{R}\right)$ and $r_{L}^{\prime}\left(r_{R}\right)$ are the phase shift and magnitudes of reflection amplitudes at the left (right) side of the chain. Eq. (1) can be derived in the framework of ballistic transport, using the Landauer-Büttiker formula [22], considering two scattering regions (namely the coupling atoms between the chain and the leads), one conducting channel in the wire and a translational invariant region within the atomic chain. The scheme of this simple model can be seen in Fig. (1). In our previous paper we investigated only symmetrical leads with one chosen orientation on the two ends of the chain and we modified the arrangement of the coupling atoms. However, in the experiments a chosen lead orientation can not be guaranteed. Therefore it is relevant to study whether the model can be applied to different lead orientations and couplings. This is the aim of the present paper.

In section 2 we describe the method we use to calculate the conductance of atomic chains placed between fcc leads. We explain how to obtain the Green's function of the leads, the Green's function of the atomic chain and how to compute the conductance of the total system. The numerical results are presented in section 3. We verify the validity of Eq. (11) by fitting the results and obtaining the reflection coefficients and the phases for systems with various leads orientations. If the ballistic model can be applied, then the fit parameters are not independent. For example by using two different symmetrical systems $\alpha \alpha$ and $\beta \beta$ (for the definitions see subsection 2.3) it is possible to predict the conductance of an asymmetrical system $\alpha \beta$ with Eq. (11). We compare this prediction with the result of the direct numerical calculation for the $\alpha \beta$ system. We finish with the conclusions. 


\section{The model}

To calculate the differential conductance of an atomic chain placed between two leads we use a tight-binding model developed by Todorov et. al. [23. The Hamiltonian, which has identical on-site energies and identical hopping energies reads

$$
\left\langle\mathbf{R}_{n}|\hat{H}| \mathbf{R}_{m}\right\rangle= \begin{cases}E_{0}, & \text { if } \mathbf{R}_{n}=\mathbf{R}_{m} \\ \gamma, & \text { if } \mathbf{R}_{n} \text { and } \mathbf{R}_{m} \text { are first neighbours } \\ 0, & \text { otherwise }\end{cases}
$$

where $\left|\mathbf{R}_{n}\right\rangle$ is the atomic state of an electron located on the $n$th atom with position vector $\mathbf{R}_{n}$. Hopping is allowed only between the first neighbors and the topology of the atom arrangements determines the physical system. In the calculations $E_{0}=0$ and $\gamma=-1$ is taken as the zero point and the unit of our energy scale. Three differently oriented blocks are investigated, i. e. the surface planes of the leads are (111), (100) and (110), so we have six different lead pairs.

The two leads are formed from semi-infinite stack of planes specified by their normal vector. The chain and the two leads are described by the Hamiltonians $\hat{H}^{s}$ and $\hat{H}^{1,2}$, respectively, and coupled by the potential $\hat{V}$. The retarded Green's function related to the total Hamiltonian $\hat{H}$ of the coupled systems is given by $\hat{G}(E)=\lim _{\varepsilon \rightarrow 0^{+}}(E-\hat{H}+i \varepsilon)^{-1}$. To calculate the conductance of the system, $g$, we start from the general expression derived by Todorov et al. [23]:

$$
g=\frac{4 \pi e^{2}}{\hbar} \operatorname{Tr}\left[\hat{\rho}_{1}\left(E_{F}\right) \hat{t}^{\dagger}\left(E_{F}\right) \hat{\rho}_{2}\left(E_{F}\right) \hat{t}\left(E_{F}\right)\right]
$$

where $\hat{\rho}_{1,2}=-\frac{1}{\pi} \operatorname{Im} \hat{G}^{1,2}$ are the density of states for lead 1 and 2 expressed via the corresponding Green's function $\hat{G}^{1,2}$ and the transfer operator $\hat{t}$ is defined by $\hat{t}(E)=\hat{V}+\hat{V} \hat{G}(E) \hat{V}$. To calculate the conductance we need then to determine the Green's functions $\hat{G}^{1}, \hat{G}^{2}$ and $\hat{G}$ for the leads and for the coupled system. The total Green's function $\hat{G}$ can be obtained from the Dyson equation

$$
\hat{G}=\hat{G}_{0}+\hat{G}_{0} \hat{V} \hat{G}
$$

where

$$
\hat{G}_{0}=\hat{G}^{1}+\hat{G}^{2}+\hat{G}^{s}
$$

and $\hat{G}^{s}$ is the Green's function of the isolated chain. In the next two subsections we present the method we used to calculate the Green's functions of the leads $\hat{G}^{1,2}$ and the chain $\hat{G}^{s}$.

\subsection{The Green's function of the leads}

An fcc crystal lead can be built up by stacking of its atomic planes. The Bloch state of an electron in one of such planes is given by

$$
|l, \mathbf{q}\rangle=\frac{1}{\sqrt{N}} \sum_{n} \exp \left(i \mathbf{q} \cdot \mathbf{R}_{n}^{l}\right)\left|\mathbf{R}_{n}^{l}\right\rangle,
$$

where $l$ denotes the $l$ th layer of the crystal, $\mathbf{q}$ is the transverse wave vector on the $l$ th layer and $\left|\mathbf{R}_{n}^{l}\right\rangle$ is the atomic state of the electron located on the $n$th atom of the $l$ th layer (having position 
vector $\mathbf{R}_{n}^{l}$ ). Here $1 / \sqrt{N}$ is a normalization factor. The leads are supposed to be semi-infinite; $l=0,1,2, \ldots \infty$. Both $\left\{\left|\mathbf{R}_{n}^{l}\right\rangle\right\}$ and $\{|l, \mathbf{q}\rangle\}$ are complete orthonormal bases. Every atom has 12 nearest neighbors. In the case of $(100)$ planes there are 4 neighbors on the same plane while $(2 \times 4)$ on the next two adjacent planes. Similarly, for (111) planes there are 6 neighbors located on the same plane and $(2 \times 3)$ on the adjacent planes. When the lead is built from (110) planes there are 2 neighbors on the same plane, and $(2 \times 4)$ on the nearest and $(2 \times 1)$ on the second nearest adjacent planes. One can calculate the dispersion relation $E(\mathbf{q})$ of the electrons on the layer as well as the coupling between layers, i.e., the matrix elements $\langle l, \mathbf{q}|\hat{H}| m, \mathbf{q}\rangle$, where $m=l, l \pm 1, l \pm 2$ depending on the orientation of the planes in the leads. To calculate the density of states that appears in Eq. (3) we need to construct the Green's functions $\hat{G}^{1,2}$ of the leads from the solution of the Dyson equation for the semi-infinite stack of the coupled planes. In the case of the orientation (111), the Green's function has already been derived by Todorov et al. [23]. In a similar way one can derive an explicit expression for the Green's function in the case of stacking of (100) planes. However, for stacking of (110) planes the method used in Ref. 23] cannot be applied because every atom has neighbors not only on the first but also on the second adjacent layers. In this case we used Haydock recursion [24 to calculate the Green's function numerically. The smoothing of the density of states is achieved by the commonly used termination of the recursion. The chain is connected to the leads in such a way, that the position of the first atom of the chain is equal to the atomic position in the next imaginary atomic plain of the lead. (Index $l$ of this fictional plain would be equal to -1.) In the (111), (100) and (110) orientations there are 3,4 and 5 connecting atoms, respectively.

\subsection{The Green's function of the atomic chain}

We now consider a one-dimensional atomic chain with $n$ atoms labelled by $s_{1}, s_{2}, \cdots, s_{n}$. In the tight-binding approximation the Hamiltonian of the chain can be written as

$$
\hat{H}^{s}=E_{0} \sum_{i=1}^{n}\left|s_{i}\right\rangle\left\langle s_{i}\right|+\gamma \sum_{i=1}^{n-1}\left(\left|s_{i}\right\rangle\left\langle s_{i+1}|+| s_{i+1}\right\rangle\left\langle s_{i}\right|\right),
$$

where $\left\{\left|s_{i}\right\rangle\right\}$ are the orthonormal atomic basis in the chain. Due to the special form of $\hat{H}^{s}$, the Green's function $\hat{G}^{s}(E)=\lim _{\varepsilon \rightarrow 0^{+}}\left(E-\hat{H}^{s}+i \varepsilon\right)^{-1}$ can easily be calculated using recursively the following identity:

$$
(A+|x\rangle\langle y|)^{-1}=A^{-1}-\frac{A^{-1}|x\rangle\langle y| A^{-1}}{1+\left\langle y\left|A^{-1}\right| x\right\rangle} .
$$

This identity is valid for arbitrary vectors $|x\rangle$ and $|y\rangle$. This method is more versatile than the one used in Ref. 23]. It can be used, e. g. for atomic chains with dangling segments or loops of atoms.

\subsection{The conductance}

The conductance given by Eq. (3) can be obtained by performing the trace in the atomic basis $\left\{\left|\mathbf{R}_{n}\right\rangle\right\}$. After straightforward manipulation we find

$$
g_{\alpha, \beta}=\frac{8 e^{2}}{h} \operatorname{Im}\left(f_{\alpha}\right) \operatorname{Im}\left(f_{\beta}\right)\left|\left\langle s_{1}\left|\hat{G}\left(E_{F}\right)\right| s_{n}\right\rangle\right|^{2}|\gamma|^{4},
$$

where $\alpha$ and $\beta$ denote the orientation of the leads 1 and 2, i. e. (100), (110) or (111) planes. Factors $f_{\alpha}$ and $f_{\beta}$ in Eq. (9) are related to the non-zero matrix elements of the density of states 
of the leads. For the three different orientations we find the following explicit forms:

$$
\begin{aligned}
f_{111}= & \frac{1}{N^{2}} \sum_{i, j=1}^{N} G_{00}^{(111)}\left(3+6 \cos \frac{2 \pi i}{N}\right), \\
f_{100}= & \frac{4}{N^{2}} \sum_{i, j=1}^{N} G_{00}^{(100)}\left(1+\cos \frac{2 \pi i}{N}+\cos \frac{2 \pi j}{N}+\cos \frac{2 \pi i}{N} \cos \frac{2 \pi j}{N}\right), \\
f_{110}= & \frac{4}{N^{2}} \sum_{i, j=1}^{N}\left[G_{00}^{(110)}\left(1+\cos \frac{2 \pi i}{N}+\cos \frac{2 \pi j}{N}+\cos \frac{2 \pi i}{N} \cos \frac{2 \pi j}{N}\right)\right. \\
& \left.+\frac{G_{11}^{(110)}}{4}+\left(G_{01}^{(110)}+G_{10}^{(110)}\right) \cos \frac{\pi i}{N} \cos \frac{\pi j}{N}\right],
\end{aligned}
$$

where $G_{l, m}^{\alpha}$ are the matrix elements of the Green's function between layers $l$ and $m$ of the $\alpha$ oriented lead. Finally, in Eq. (9) $\left\langle s_{1}\left|\hat{G}\left(E_{F}\right)\right| s_{n}\right\rangle$ are the matrix elements of the total Green's function of the system between the first and the last atoms of the chain. Using the Dyson equation (41) we obtain

$$
\left\langle s_{1}\left|\hat{G}\left(E_{F}\right)\right| s_{n}\right\rangle=\frac{G_{1 n}^{s}}{1-\left(G_{11}^{s} f_{\alpha}-G_{n n}^{s} f_{\beta}\right)|\gamma|^{2}+D\left(G^{s}\right) f_{\alpha} f_{\beta}|\gamma|^{4}},
$$

where $D\left(G^{s}\right)=\left(G_{11}^{s} G_{n n}^{s}-G_{1 n}^{s} G_{1 n}^{s}\right)$ with matrix elements $G_{i j}^{s}=\left\langle s_{i}\left|\hat{G}^{s}\right| s_{j}\right\rangle$ of the Green's function for the atomic chain. We now have all the necessary formulas to calculate numerically the conductance.

\section{Numerical results}

The dispersion relation of the chain in tight-binding approximation reads

$$
E(k)=E_{0}+2 \gamma \cos (k a),
$$

where $a$ is the lattice constant. Thus, the chain has states only in the $E_{0}-2 \gamma \ldots E_{0}+2 \gamma$ interval therefore this is the energy range where the system can work as a conductor. We have taken 9 different energy points in this interval and calculated the conductance with 6 different lead pairs. As a first step we checked the validity of the ballistic transport model by doing some tests. We fitted Eq. (11) on the numerical results to check whether the required relations between the parameters are fulfilled or not. For the phase $\Phi_{L R}$ in Eq. (1) there holds an addition rule. The phase of the conductance oscillations of a chain between $\alpha$ and $\beta$ leads is related to the phases of the oscillations between the symmetrical $(\alpha \alpha$ and $\beta \beta)$ leads as

$$
\Phi_{\alpha \beta}=\frac{\Phi_{\alpha \alpha}+\Phi_{\beta \beta}}{2} .
$$

This relation is fulfilled by our numerical results with an accuracy better than $1 \%$. The values of the phases from the fit of Eq. (1) are summarized in Table 1, The good agreement is clearly visible at every energy level. The energy dependence of the phases can be seen in Fig. 2, For symmetrical leads $r_{L}^{\prime}=r_{R}$, so these parameters can be determined also from the fit as it can be seen in Table 1 and in Fig. 3 ,

Another proof of the validity of Eq. (1) can be obtained as follows: By calculating the conductance of two different but symmetrical systems, for example between $\alpha \alpha$ and $\beta \beta$ leads, with 
6P. Major, G. Tichy, J. Cserti, V. M. García-Suárez, and S. Sirichantaropass: The geometry dependence of the conductance oscillati

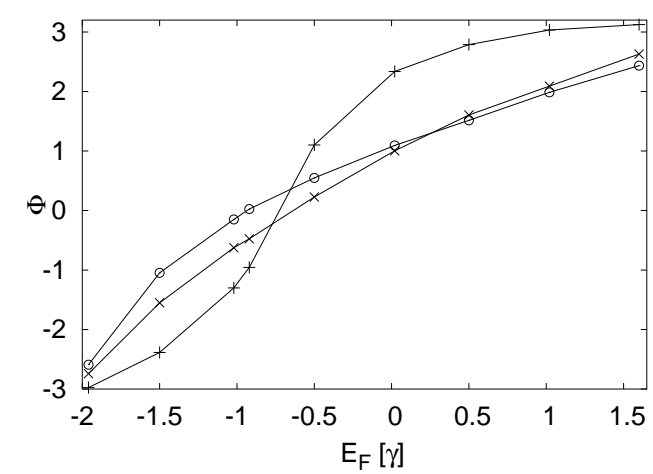

Fig. 2 The energy dependence of the phases $\Phi_{L R}$ for symmetrical leads. Signs,$+ \times$ and $\circ$ denote the orientations (111), (100) and (110), respectively.

the fitted parameters, $\Phi_{\alpha \alpha}, \Phi_{\beta \beta}, r_{\alpha}, r_{\beta}$, the conductance of the asymmetric $\alpha \beta$ system can be predicted. To do this we need to substitute $\Phi_{\alpha \beta}$ from Eq. (13), $r_{L}^{\prime}=r_{\alpha}$ and $r_{R}=r_{\beta}$ in Eq. (11). The comparison of the predicted and the directly calculated results is plotted in Fig. 4 and implies that differently oriented leads do not modify the availability of Eq. (1). The orientation of the leads influences the conductance essentially due to the different chain-lead coupling and different density of the atoms on the surface. The above results suggest that all of these properties can be described by the parameters $r_{L}^{\prime}, r_{R}$ and $\Phi_{L R}$, and we do not need to modify the simple ballistic transport model for different leads.

We found that the wavelength of the oscillations does not depend on the length and the character of the leads. It is essentially determined by the dispersion relation of the chain, similarly to our earlier results [17]. At half filled band the wavenumber is $\pi /(2 a)$, which gives two-atom-periodicity, but at other Fermi levels it becomes different. For example, for wavenumbers $k=\pi /(4 a)$ or $k=(3 \pi) /(4 a)$ it yields four-atom-periodic oscillations, in agreement with the results of Thygesen

Table 1 The phase $\Phi$ and reflection amplitude $r$ of the oscillations at different energies, resulted from the fit of Eq. (1).

\begin{tabular}{c|cccccc|ccc}
$E[\gamma]$ & $\Phi_{111,111}$ & $\Phi_{100,100}$ & $\Phi_{110,110}$ & $\Phi_{111,100}$ & $\Phi_{111,110}$ & $\Phi_{100,110}$ & $r_{111,111}$ & $r_{100,100}$ & $r_{110,110}$ \\
\hline-1.96 & -2.976 & -2.741 & -2.591 & -2.861 & -2.781 & -2.661 & 0.651 & 0.706 & 0.712 \\
-1.5 & -2.386 & -1.551 & -1.047 & -1.968 & -1.716 & -1.299 & 0.199 & 0.314 & 0.368 \\
-1.02 & -1.300 & -0.626 & -0.151 & -0.972 & -0.745 & -0.391 & 0.085 & 0.233 & 0.311 \\
-0.92 & -0.955 & -0.476 & 0.024 & -0.696 & -0.446 & -0.226 & 0.071 & 0.224 & 0.309 \\
-0.5 & 1.101 & 0.227 & 0.547 & 0.660 & 0.810 & 0.395 & 0.067 & 0.206 & 0.307 \\
0.02 & 2.336 & 1.004 & 1.093 & 1.667 & 1.718 & 1.042 & 0.114 & 0.213 & 0.323 \\
0.5 & 2.787 & 1.605 & 1.515 & 2.200 & 2.140 & 1.560 & 0.177 & 0.244 & 0.353 \\
1.02 & 3.033 & 2.090 & 1.985 & 2.615 & 2.440 & 2.070 & 0.272 & 0.315 & 0.415 \\
1.6 & 3.124 & 2.629 & 2.434 & 2.869 & 2.779 & 2.544 & 0.456 & 0.479 & 0.559
\end{tabular}




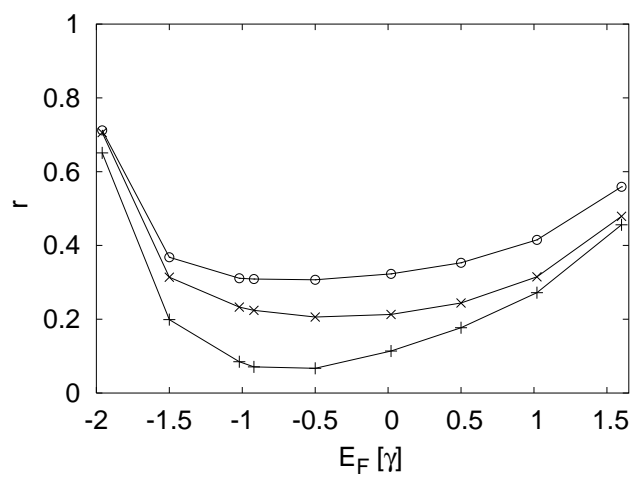

Fig. 3 The energy dependence of the reflection coefficients $r$ for symmetrical leads. Signs,$+ \times$ and $\circ$ denote the orientations (111), (100) and (110), respectively.

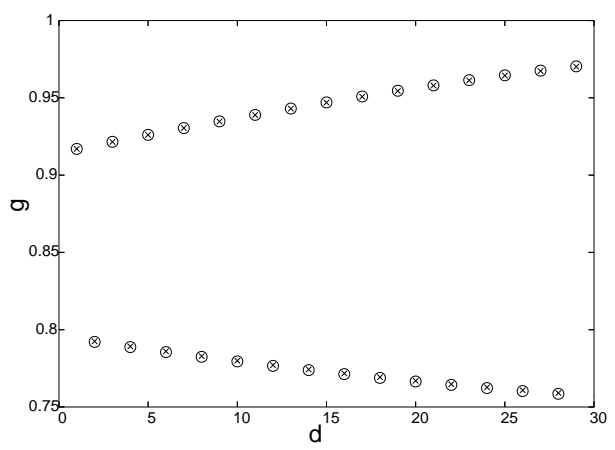

Fig. 4 The conductance (in units of $2 e^{2} / h$ ) of a chain between asymmetric $(\alpha \beta)$ leads, at energy $E_{F}=0.02 \gamma$ determined by Eq. (1), substituting the parameters of the symmetrical $\alpha \alpha$ and $\beta \beta$ systems (crosses), and that from direct calculation (open circles) as a function of the length $d$ (in units of the lattice constant $a$ ) for lead orientations $\alpha=(110)$ and $\beta=(100)$.

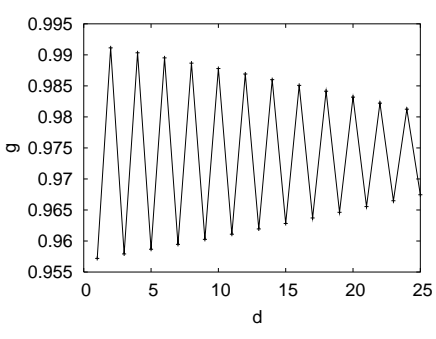

a

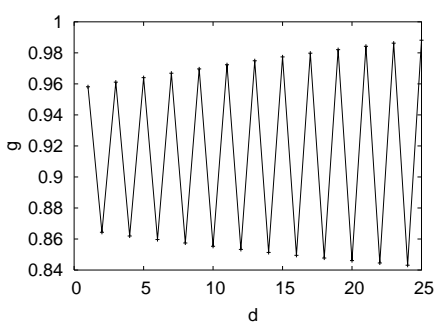

$\mathrm{b}$

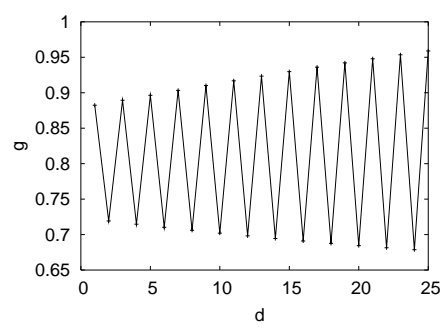

C

Fig. 5 The conductance (in units of $2 e^{2} / h$ ) as a function of the length $d$ (in units of the lattice constant a) of the chain between symmetrical leads at $E_{F}=0.02 \gamma$. Figs. a, b and c show the results for the lead orientations 111-111, 100-100 and 110-110, respectively.

and Jacobsen [25], who found that the three conductance channels of aluminium have wavenumbers $\pi /(4 a), \pi /(2 a)$, and $(3 \pi) /(4 a)$, and the conductance oscillations have four atoms periodicity. The periodicity of the oscillations given by Eq. (1) is also in agreement with the earlier prediction of de 
la Vega and co-workers [26, who predicted that every conduction channel oscillates as a function of length with a wavenumber $2 k_{F}$.

For monovalent atomic chains the charge neutrality condition requires wavenumber $k_{F}=$ $\pi /(2 a)$. Then, it follows from Eq. (11) that the conductance is an alternating function of the number of atoms. At $k_{F}=\pi /(2 a)$ the phase can not be determined easily from the fit on numerical data and it seems to be an unnecessary parameter. Maybe this fact can explain why the phase was missed in earlier interpretation models [5, 6, 10, 15, 16. However, in spite of these difficulties the phase is very useful to interpret the geometry dependence of the oscillations. To avoid numerical problems, we could take the $\operatorname{limits} \lim _{E \rightarrow E_{F}} \Phi(E)$ and $\lim _{E \rightarrow E_{F}} r(E)$, but as can be seen in Figs. 2 and 3 these parameters are smooth functions of the energy and therefore we choose $E_{F}=0.02 \gamma$ in the calculations. For symmetrical leads some numerical results are plotted in Fig. 5. Because of the different groups of atoms connecting the chain to the lead, the oscillations have different phases. Between leads with planes (100) and (110) one can see that the conductance for chains with an even atoms is smaller than for chains with an odd number of atoms, i. e. $e<o$. However, in the case of (111) orientation, the so called even-odd effect changes its sign and $e>o$. This demonstrates the sensitive geometry dependence of the conductance. The analysis of Eq. (1) implies that the maximum value of the conductance can be $\left(2 e^{2} / h\right)$ only if the cosine term is equal to 1 and therefore the phase is 0 . It is also clear from the figure that not only the phases, but also the amplitudes depend on the coupling, since according to Eq. (11) larger reflection coefficient $r$ yields larger amplitude of the oscillation. Indeed we have found the relation $r_{111}<r_{100}<r_{110},\left(0.114,0.213\right.$ and 0.323 , respectively at $\left.E_{F}=0202 \gamma\right)$ which follows the same pattern as the amplitudes of the oscillations. In the case of orientations (111), (100), (110) there are 3, 4 and 5 coupling atoms between the lead and the chain, respectively, suggesting that more atoms may increase the reflection amplitudes. However, it is also necessary to stress that the different chain-lead couplings result in different phases, and these can modify the amplitudes of the oscillations also.

\section{Conclusions}

We have shown that the differently oriented leads do not affect the applicability of Eq. (1). However, the geometrical arrangement of the atoms connecting the chain to the lead appreciably influences the conductance, since the phases and the amplitudes of the oscillations change significantly and the so-called even-odd effect can change its sign. This implies that numerical results of different calculation methods are comparable only in the case of equal geometrical circumstances. The validity of Eq. (11) can advance further investigations of the geometry dependence of the oscillations, since it can be used to predict the transport characteristics of asymmetrical leads, so that it would only be necessary to perform simulations of symmetrical systems.

Since the parameters of the oscillations depend so sensitively on the details of the coupling between the chain and the leads, it is not clear at first sight why the even-odd effect can be detected experimentally. A possible explanation is the following. In the experiments the average of conductances of several different contacts is measured. The different geometries and consequently oscillations with different phases could suppress the even-odd effect. However, there are energetically preferred surface orientations for a chosen material, and these facets assist the formation of preferred chain-lead couplings, resulting favored certain $r$ and $\Phi$ parameter values. Rodrigues et al. 27, 28 investigated the effect of these preferred surfaces on the conductance of point contacts experimentally, and they found that in the case of gold the (111) facets are more favorable. An other argument can be the following. In the experiments after one breaking of the contact the tips are brought together again to form a new contact. The displacement and variation of both parts are usually small. Therefore the recreated contact has similar geometry and presumably similar oscillation parameters. 
In summary we showed that Eq. (11) and specially its phase plays an important role to interpret the conductance oscillations of atomic wires of monovalent elements.

Acknowledgements The authors would like to thank the helpful discussions with Gergely Szirmai and Tamás Herpay. VMGS thanks the European Union for a Marie Curie grant.

\section{References}

[1] Hideaki Ohnishi, Yukihito Kondo, Kunio Takayanagi, Nature 395780 (1998)

[2] A. I. Yanson, G. R. Bollinger, H. E. van den Brom, N. Agraït, J. M. van Ruitenbeek, Nature 395, 783 (1998);

[3] P. L. Pernas, A. Martín-Rodero, and F. Flores, Phys. Rev. B 41, R8553 (1990).

[4] N. D. Lang, Phys. Rev. Lett. 79, 1357 (1997).

[5] R. H. M. Smit, C. Untiedt, G. Rubio-Bollinger, R. C. Segers, J. M. van Ruitenbeek, Phys. Rev. Lett. 91, 076805 (2003).

[6] H.-S. Sim, H.-W. Lee, and K. J. Chang, Phys. Rev. Lett. 87, 096803 (2001).

[7] R. Gutiérrez, F. Grossmann, and R. Schmidt, Acta Phys. Pol. B 32, 443 (2001).

[8] S. Tsukamoto and K. Hirose, Phys. Rev. B 66, 161402(R) (2002).

[9] Y. Egami, T. Ono, and K. Hirose, Phys. Rev. B 72, 125318 (2005).

[10] Y. J. Lee, M. Brandbyge, M. J. Puska, J. Taylor, K. Stokbro, and R. M. Nieminen, Phys. Rev. B 69, 125409 (2004).

[11] H.-W. Lee and C.-S. Kim, Phys. Rev. B 63, 075306 (2001).

[12] Z. Y. Zeng and F. Claro, Phys. Rev. B 65, 193405 (2002).

[13] P. Havu, T. Torsti, M. J. Puska, R. M. Nieminen, Phys. Rev. B 66, 075401 (2002)

[14] K. Hirose, N. Kobayashi, and M. Tsukada, Phys. Rev. B 69, 245412 (2004).

[15] E. G. Emberly and G. Kirczenow, Phys. Rev. B 60, 6028 (1999).

[16] H.-W. Lee, H.-S. Sim, D.-H. Kim, and K. J. Chang, Phys. Rev. B 68, 075424 (2003).

[17] P. Major, V. M. García-Suárez, S. Sirichantaropass, J. Cserti, C. J. Lambert, J. Ferrer, G. Tichy Phys. Rev. B 73045421

[18] Recent experiments have also studied the interaction of atomic chains with simple molecules; Sz. Csonka, A. Halbritter and G. Mihály, Phys. Rev. B 73, 075405 (2006); W. H. A. Thijssen, D. Marjenburgh, R. H. Bremmer, and J. M. van Ruitenbeek Phys. Rev. Lett. 96, 026806 (2006).

[19] A. R. Rocha, V. M. García-Suárez, S. W. Bailey, C. J. Lambert, J. Ferrer, and S. Sanvito, Phys. Rev. B 73, 085414 (2006).

[20] A. R. Rocha, V. M. García-Suárez, S. W. Bailey, C. J. Lambert, J. Ferrer and S. Sanvito, Nature Materials 4, 335 (2005).

[21] V. M. Garía-Suárez, A. R. Rocha, S. W. Bailey, C. J. Lambert, S. Sanvito, and J. Ferrer, Phys. Rev. Lett. 95, 256804 (2005).

[22] R. Landauer, IBM J. Res. Dev. 1, 223 (1957); M. Büttiker, Phys. Rev. Lett. 57, 1761 (1986); S. Datta, Electronic Transport in Mesoscopic Systems, (Cambridge University Press, Cambridge, 1995).

[23] T. N. Todorov, G. A. D. Briggs, and A. P. Sutton J. Phys. Condens. Matter 5, 2389 (1993); T. N. Todorov, Phys. Rev. B 54, 5801 (1996).

[24] R. Haydock, The recursive Solution of the Schrödinger equation in Solid State Physics edited by H. Ehrenreich, F.Seitz, and D. Turnbull, vol 35, pages 215-294 Academic press, New York, 1980; R. Haydock, V. Heine, and M. J. Kelly, J. Phys. C 5, 2845 (1972); [INSPEC] ibid. 8, 2591 (1975); [INSPEC]; C. Lanczos, J. Res. Natl. Bur. Stand. 45, 225 (1950).

[25] K. S. Thygesen and K. W. Jacobsen, Phys. Rev. Lett. 91, 146801 (2003).

[26] L. de la Vega, A. Martín Rodero, A. Levy Yeyati, A. Saúl Phys. Rev. B 70, 113107 (2004)

[27] V. Rodrigues, J. Bettini, A. R. Rocha, L. G. C. Rego, D. Ugarte, Phys. Rev. B 65, 153402 (2002)

[28] V. Rodrigues, T. Fuhrer, D.Ugarte, Phys. Rev. Lett. 854124 (2000) 